\title{
Battery Management System for Electric Vehicle and the Study of SOC Estimation
}

\author{
Xueqing Yuan, Lin Zhao, Bo Li, Naiming Liu \\ Shenyang Institute of Automation Chinese Academy of Science, No. 19, Feiyun Road, Hunnan New District, Shenyang City, \\ Liaoning Provence, China, 110179
}

\begin{abstract}
The SOC (state of charge) of the Li-ion battery cells in a pack are different because of the property differences, which would lead to over-charging/overdischarging the battery pack and as a result the service life of the battery pack would be reduced. In this article, we designed a battery management system (BMS) for low voltage electric vehicle. The BMS adopted resistance shunt method to avoid over-charging the battery cells. Extended Kalman filter (EKF) was utilized for high precision estimation of SOC, which is very important for remaining the cells working within appropriate SOC and avoiding over-discharging the cells. Experiment result shows that comparing with the commonly used ampere-hour integration approach, EKF decreased estimation error from $15.48 \%$ to $7.27 \%$. High precision SOC estimation algorithm and effective charge equalization method can maintain the battery cells working at a good situation and extend the service life of the battery pack, reducing the cost of use indirectly. This is meaningful for Li-ion battery's industrial application.
\end{abstract}

\section{Keywords-BMS; charge equalization; SOC Estimation.}

\section{INTRODUCTION}

As a newly-developing storage battery, lithium iron phosphate battery has been more and more widely used in different industrial fields such as electric vehicle, intelligent power grid energy storage, electronic instrument and so on, working as power supply system, for its advantages of no pollution, high energy density and security[1]. As a single cell usually cannot supply enough voltage and power, Li-ion battery is often used in packs.
However, this could lead to over-charge or over-discharge for the individual property differences of the cells and the differences of state of charge (SOC), which would reduce the battery stack's service life, indirectly increasing the application cost[2][3]. Therefore, each battery stack should be equipped with a battery management system (BMS) which has the functions of SOC estimation, overcharge/over-discharge protection and charge equalization, to ensure that the battery stack can work efficiently and securely.

In this paper, we designed a BMS for low-voltage electric vehicles. The controller of the system is a micro control unit (MCU) whose version is MC9S12XEQ384 designed by Freescale company. Passive equalization method is adopted for charge/discharge equalization, and extended Kalman filter (EKF) is adopted as the SOC estimation algorithm which is a nonlinear identification method with high precision and the SOC estimation error is only $7.27 \%$. The system is confirmed having an excellent effect on maintaining battery stacks and ensuring safety in using the low-voltage electric vehicle.

\section{HARDWARE STRUCTURE OF THE BMS}

The BMS introduced in this article is designed for low-voltage electric vehicle, and its output voltage is $48 \mathrm{~V}$, composed with 16 cells connected in series whose nominal voltage is $3 \mathrm{~V}$. The main function of the BMS is to monitor and maintain the energy system of the electric mobile. The structure of the BMS is shown in Figure 1.

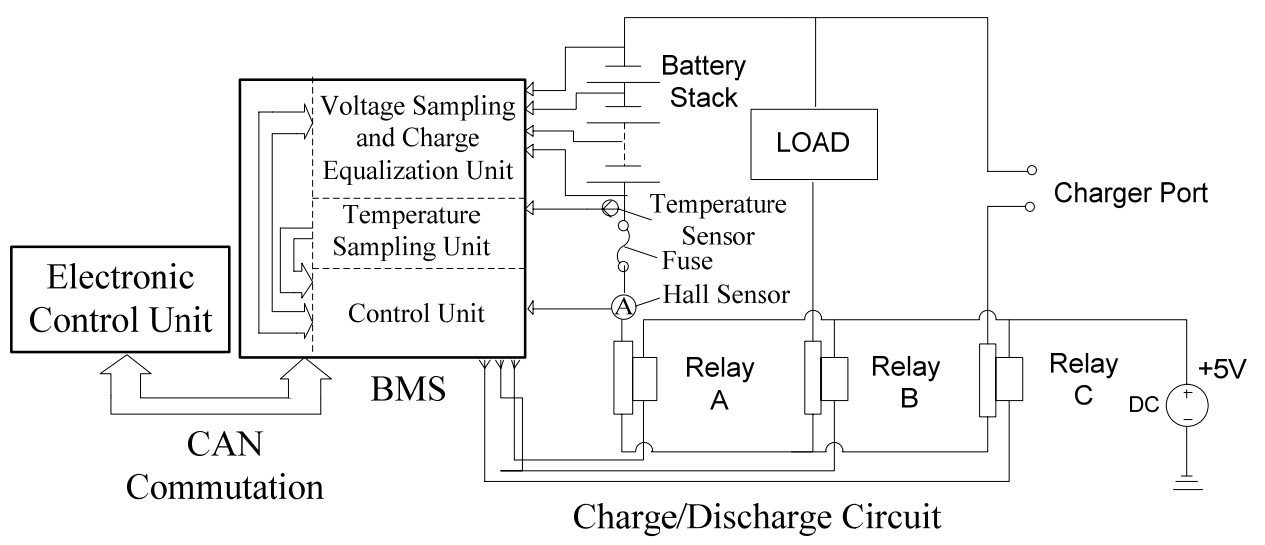

Figure 1. Power System of the Electric Vehicle 
In Figure 1, the power system of the electric vehicle is composed of BMS, charge/discharge circuit and a battery stack. BMS is the control center of the whole system, controlling the charge/discharge circuit through relay A, B, and $\mathrm{C}$, monitoring voltage, current, temperature of the battery stack through measurement units, and communicating with the electronic control unit (ECU) through CAN communication. When the relay $\mathrm{A}$ and $\mathrm{B}$ connects, the battery stack supply power for the electric vehicle, and when the relay $\mathrm{A}$ and $\mathrm{C}$ connects, the charger charges the battery stack. The BMS has many measurement units. Temperature sampling unit monitors the battery stack's temperature through temperature sensor, and if the battery stack's temperature is out of the safety range resulting from overcurrent or other faults of the system, BMS would shut down the whole circuit to avoid permanent damage of the battery stack or even explosion accidents. Voltage sampling unit measures each cell's voltage and the battery stack's total voltage, and BMS implements over-charge/over-discharge protect and charge equalization through the voltage information. Current sampling unit measures the current through the Hall sensor allocated in the charge/discharge circuit. Current information is the most important signal to estimate the battery stack's SOC. In the positive pole a fuse is allocated to shut down the circuit in case that the current surges because of short circuit or other faults. As the battery stack is only composed of 16 cells, all the units of the BMS is integrated in one circuit board, and the structure of the circuit board is shown in Figure 2.

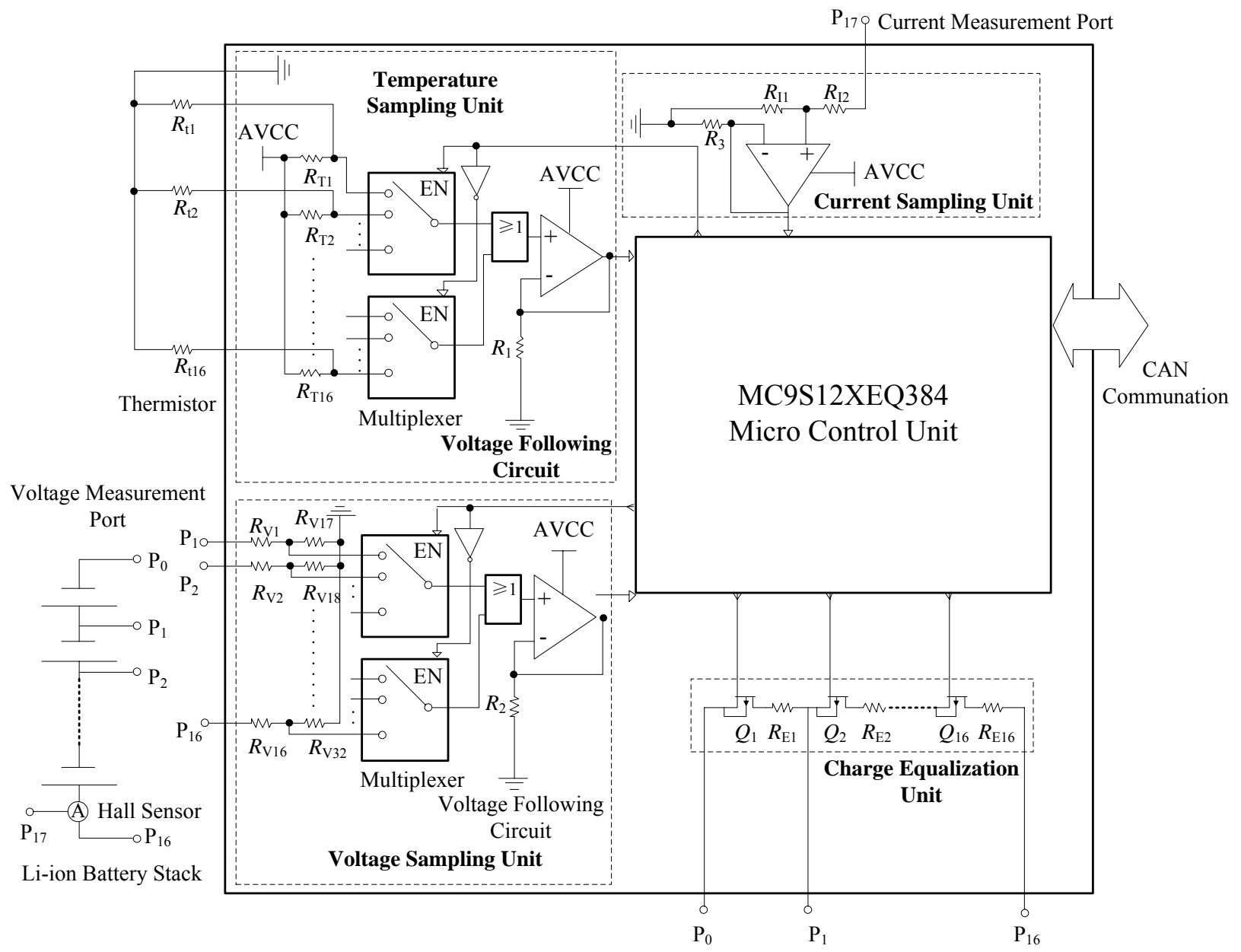

Figure 2. Hardware Structure of BMS

In Figure 2, MC9S12XEQ384 MCU controls and deploys temperature sampling unit, current sampling unit, voltage sampling unit and charge equalization unit. As MC9S12XEQ384 has a 16 bit AD converter inside, all the sampled analog signals were directly transferred into MC9S12XEQ384. The voltage and temperature signals go through voltage division circuit, a multiplexer, voltage following circuit, and at last into the MCU. The measurement port of the voltage sampling unit connects with the cells in series. The thermistors of the temperature sampling unit contact directly with the cells, to accurately measure the temperatures of the cells. Current signal go 
through voltage division circuit, a multiplexer and then into the MCU. Resistance-shunt method is applied in the charge equalization unit. The shunt resistances are connected in parallel with the cells, and the shunt current is controlled through setting the duty ratio of the switches $\mathrm{Q}_{1} \sim \mathrm{Q}_{16}$. The MCU implements the operations of signal reading, SOC estimation and the control of the whole system through the programs written inside, which will be introduced in next section.

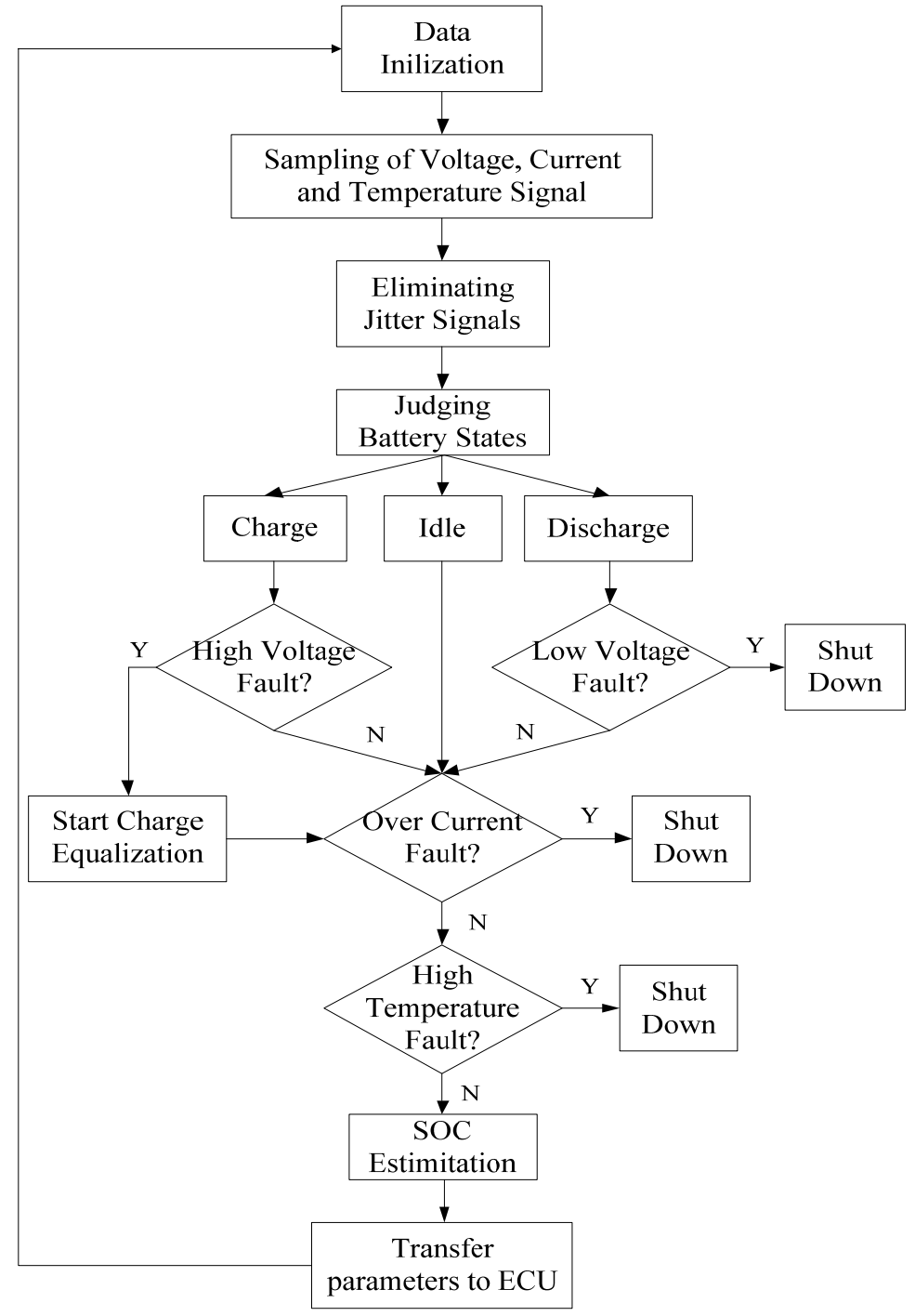

Figure 3. Software Flow Pattern of BMS

\section{Program StRUCTURE OF BMS}

The BMS designed in this article is programed in $\mathrm{C}$, and the flow chart is shown in Figure 3. Hardware interface program is generated automatically by the expert system of the software "Code Warrior". In the program of MCU, the functions of reading voltage, current and temperature signals, anti-jitter of the current signal, the protection of over-charge/over-discharge, over current, high/low temperature, charge equalization and SOC estimation is implemented. The method of anti-jitter of the current signal is selecting the signals which is out of the range of the threshold set beforehand, eliminating it and computing the signal in that moment by smoothing the signals a moment before. We apply the method in reference [4] in charge equalization. Once one of the faults of low voltage, over current and high temperature happens, the MCU would shut down the whole circuit to avoid permanent damages of the battery stack or even explosion events. SOC estimation is one of the most difficult parts of BMS design, and we will introduce it in next section in detail.

Once the BMS starts to work, it recycles the program in Figure 3 until abnormal conditions occurred and the 
MCU would shut down the whole circuit to protect the battery stack.

\section{The SOC Estimation Method BAsed ON EKF}

SOC estimation of the battery stack is one of the most important functions of BMS, the base to maintain the battery stack working within reasonable range, and also one of the most difficult parts in BMS design. The most widely used SOC estimation method is ampere-hour integration approach[5][6]. Its principle is computing out the change of the charge by integrating current, adding the changed change to the initial charge and the whole charge can be computed out. This method is easy to implement, however, its estimation precision is often not satisfying and will divergence with time. In this article, we apply EKF in the SOC estimation, which is a nonlinear estimation method with high precision. In this method, the SOC of the battery stack is a state variable, and the state equalization is set up upon the battery model and the basic principle of ampere-hour integration approach. The battery model selected in this article is shown in Figure 4.

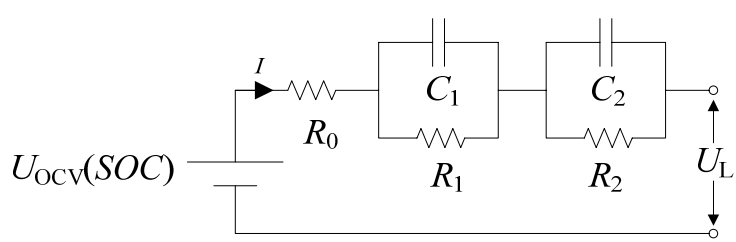

Figure 4. Li-ion Battery Model

In Figure $4, R_{0}$ is the internal resistance of a cell, $R_{1} C_{1}$, $R_{2} C_{2}$ is two RC loops to simulate the polarity effect of a cell. $U_{\mathrm{OCV}}(S O C)$ is the open circuit voltage of a cell, which is the function of the battery SOC. The parameters of a cell above can be measured through hybrid pulse power characteristic (HPPC) experiment. Set the voltages of $R_{1} C_{1}, R_{2} C_{2}$ separately is $U_{1}, U_{2}$, and the current is $I$, the relationship between voltage and current of the RC loop is

$$
\begin{aligned}
& \frac{d U_{1}}{d t}=-\frac{1}{R_{1} C_{1}} U_{1}+\frac{1}{C_{1}} I \\
& \frac{d U_{2}}{d t}=-\frac{1}{R_{2} C_{2}} U_{2}+\frac{1}{C_{2}} I
\end{aligned}
$$

Define time constant $\tau_{1}=R_{1} C_{1}, \tau_{2}=R_{2} C_{2}$, then

$$
\begin{aligned}
& U_{\mathrm{OCV}}(S O C)=I R_{0}+U_{1}+U_{2} \\
& U_{1}=U_{1}(0) e^{-\frac{t}{\tau_{1}}}+I R_{1}\left(1-e^{-\frac{t}{\tau_{1}}}\right) \\
& U_{2}=U_{2}(0) e^{-\frac{t}{\tau_{2}}}+I R_{2}\left(1-e^{-\frac{t}{\tau_{2}}}\right)
\end{aligned}
$$

Where $U_{1}(0), U_{2}(0)$ is the initial voltage of capacitance $C_{1}, C_{2}$. Most SOC estimation methods based on EKF adopt 3-rank polynomial to match OCV-SOC curve, which has a big error. In this article, we adopt 6-rank polynomial and an exponential function to match OCVSOC curve, which improves the matching precision remarkably:

$$
U_{\mathrm{OCV}}(S O C)=a_{0}+a_{1} S O C+\cdots+a_{6} S O C^{6}+b_{0} e^{b_{1} S O C}
$$

The parameters $a_{0}, a_{1} \ldots a_{6}, b_{0}, b_{1}$ only relate to the battery character. According to reference [7], the open circuit voltage $(\mathrm{OCV})$ can be measured quickly, and the parameters can be estimated by least squared method according to the OCV-SOC curve. The sampling time is denoted $\Delta \mathrm{t}$, and the electric quantity denoted $Q_{0}$, then the relationship between SOC, current and electric quantity is

$$
\operatorname{SOC}(k+1)=\frac{Q(k+1)}{Q_{0}}=\frac{Q(k)+I \Delta t}{Q_{0}}=\operatorname{SOC}(k)+\frac{\Delta t}{Q_{0}} I
$$

Where $k$ denotes sampling time. Any nonlinear system state equation can be written as

$$
\begin{aligned}
& X(k)=A X(k-1)+B U(k-1)+W(k) \\
& Z(k)=g(X(k), U(k))+V(k)
\end{aligned}
$$

Where $X$ denotes state variable, $U$ denotes input signal, $Z$ denotes measured output signal, $W$ and $V$ separately denotes driving noise and measured noise, which are not uncorrelated white noises, whose variance matrix is separately denoted $Q$ and $R$.

Combining with equation(2), the system state equation of the cell can be written as

$$
\begin{aligned}
& {\left[\begin{array}{c}
\operatorname{SOC}(k+1) \\
U_{1}(k+1) \\
U_{2}(k+1)
\end{array}\right]=\left[\begin{array}{ccc}
1 & 0 & 0 \\
0 & e^{-\Delta t / \tau_{1}} & 0 \\
0 & 0 & e^{-\Delta t / \tau_{2}}
\end{array}\right]\left[\begin{array}{c}
S O C(k) \\
U_{1}(k) \\
U_{2}(k)
\end{array}\right]+\left[\begin{array}{c}
\Delta t / Q_{0} \\
R_{1}\left(1-e^{-\Delta t / \tau_{1}}\right) \\
R_{2}\left(1-e^{-\Delta t / \tau_{2}}\right)
\end{array}\right] I(k)+W(k)} \\
& U_{\mathrm{L}}(k)=U_{\mathrm{OCV}}(\operatorname{SOC}(k))+I(k) R_{0}+U_{1}(k)+U_{2}(k)+V(k)
\end{aligned}
$$

Where the state variable is $X(k)=\left[S O C(k), U_{1}(k)\right.$, $\left.U_{2}(k)\right]^{\mathrm{T}}$, and the input signal is $U(k)=I(k)$, measured output
$Z(k)=U_{\mathrm{L}}(k)$, and the matrix $A=\left[\begin{array}{ccc}1 & 0 & 0 \\ 0 & e^{-\Delta t / \tau_{1}} & 0 \\ 0 & 0 & e^{-\Delta t / \tau_{2}}\end{array}\right]$, 
$B=\left[\begin{array}{c}\Delta t / Q_{0} \\ R_{1}\left(1-e^{-\Delta t / \tau_{1}}\right) \\ R_{2}\left(1-e^{-\Delta t / \tau_{2}}\right)\end{array}\right]$. Matrix $C(k)$ can be computed out through

$$
C(k)=\frac{\partial g(X(k), U(k))}{\partial X(k)}=\left[\frac{d U_{\mathrm{OCV}}(S O C(k))}{d S O C(k)}, 1,1\right]
$$

Basing on the recursive algorithm of EKF, the SOC of the battery stack can be estimated with the following process:

Step1: The prediction of the system state at time $\mathrm{k}$ is $X(k \mid k-1)=A X(k-1 \mid k-1)+B U(k-1)$

Step2: The prediction of the covariance matrix of the state error is $P(k \mid k-1)=A P(k-1) A^{\mathrm{T}}+Q$
Step3: The measuring-state transfer matrix is $C(k)=\frac{\partial g(X(k), U(k))}{\partial X(k)}$

Step4:The gain matrix $K_{\mathrm{g}} \quad$ is $\quad K_{\mathrm{g}}(k)=P(k \mid k-$ 1) $C(k)\left[C(k) P(k \mid k-1) C(k)^{\mathrm{T}}+R\right]^{-1}$

Step5:The optimal estimation of the system state at time $k$ is $X(k \mid k)=X(k \mid k-1)+K_{\mathrm{g}}(k)[Z(k)-g(X(k), U(k))]$

Step6: The covariance matrix of the state error at time $k$ is $P(k \mid k)=\left[1-K_{\mathrm{g}}(k) C(k)^{\mathrm{T}}\right] P(k \mid k-1)$

Discharging a Li-ion cell with a nominal voltage of $3 \mathrm{~V}$, and estimating its SOC utilizing ampere-hour integration approach and EKF separately according the current and voltage information, the estimation error of ampere-hour integration approach is $15.48 \%$, and the EKF method is $7.27 \%$. The SOC curve is shown in Figure 5.

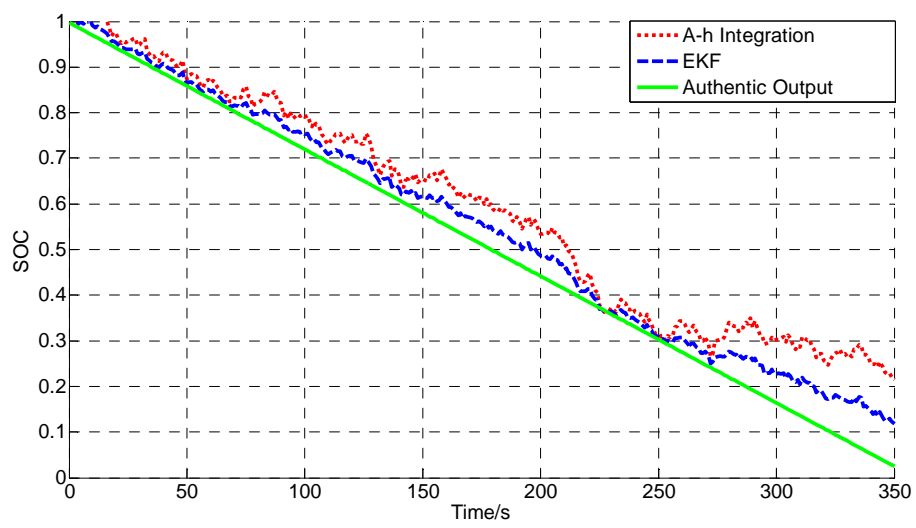

Figure 5. Experiment Result

It is showed clearly that by utilizing EKF method, the SOC estimation precision is improved remarkably comparing with ampere-hour integration approach.

\section{CONCLUSIONS}

In this article, a BMS designed by our team was introduced which can monitor voltage, current and temperature of the battery stack and protect it from overcharge/over-discharge, over current, high temperature faults according to the measured signals, and it also can estimate the SOC of the battery stack with a high precision with the improved algorithm basing on EKF. The system has been tested that it can maintain the battery stacks at a good state and improve the reliability and security of the electric vehicle.

\section{ACKNOWLEDGEMENTS}

This work was supported by Science and Technology Project of Shenyang(F12-017-2-00).

\section{REFERENCES}

[1] Hang G. X., Han J. D.. Research progress on equalization charging of Li-ion battery[J]. Chinese Battery Industry, Feb. 2009, Vol 14, No. 1, pp:65-68.

[2] Park H-S, Kim C-H. Design of a charge equalizer based on battery modularization[J]. IEEE Trans. on Vehicular Technology, 2009, Vol. 58, No. 7, pp:3216-3223.

[3] Hurley W. G., Wong Y. S., Wölfle W. H.. Self-equalization of cell voltages to prolong the life of VRLA batteries in standby applications[J]. IEEE Trans. on Industry Electronics, 2009, Vol. 56, No. 6, pp:2115-2120.

[4] Xu Shungang, Wang Jinping, Xu Jianping. An Equalizing Charge Control Strategy to Extend Battery Cycle Life for Electric Vehicles [J]. Proceedings of the CSEE, Jan. 2012, Vol. 32, No. 3, pp:43-47

[5] Li Zhe, Lu Languang. Comparison of methods for improving SOC estimation accuracy through an ampere-hour integeration approach [J]. Tsing hua Univ(Sci\&Tech), 2010, Vol. 50, No. 8, pp:12931296.

[6] Miyamoto H, Morimoto M, Morita K. On-line SOC estimation of battery for wireless tram car [C]. Proceedings of 2007 International Conference on Power Electronics and Drive Systems. Bangkok, Thailand, 2007.

[7] Suleiman Abu-Sharkh, Dennis Doerffel. Rapid test and non-linear model characterization of solid-state lithium-ion batteries [J]. Journal of Power Source, 2004, (130):266-274 